\title{
A novel approach to gravitation from fluid theory: \\ Titius-Bode structures, flat rotation rate of galaxies, and other predictions
}

\author{
Hector A. Munera \\ Centro Internacional de Fisica, Apartado aereo 4948, \\ Bogota, Colombia \\ email: hmunera@hotmail.com
}

\begin{abstract}
Following the discovery of quantum phenomena at laboratory scale (Couder \& Fort 2006), de Broglie pilot wave theory (De Broglie 1962) has been revived under a hydrodynamic guise (Bush 2015). Theoretically, it boils down to solving the transport equations for the energy and linear momentum densities of a postulated fundamental fluid in terms of classical wave equations, which inherently are Lorentz-invariant and scale-invariant. Instead of the conventional harmonic solutions, for astronomical and gravitational problems the novel solutions for the homogeneous wave equation in spherical coordinates are more suitable (Munera et al. 1995, Munera \& Guzman 1997, and Munera 2000). Two groups of solutions are particularly relevant: (a) The inherently-quantized helicoidal solutions that may be applicable to describe spiral galaxies, and (b) The non-harmonic solutions with time $(t)$ and distance $(r)$ entangled in the single variable $q=C t / r$ ( $C$ is the two-way local electromagnetic speed). When these functions are plotted against $1 / q$ they manifestly depict quantum effects in the near field, and Newtonian-like gravity in the far-field. The near-field predicts quantized effects similar to ring structures and to Titius-Bode structures, both in our own solar system and in exoplanets, the correlation between predicted and observed structures being typically larger than 99 per cent. In the far-field, some non-harmonic functions have a rate of decrement with distance slower than inverse-square thus explaining the flat rotation rate of galaxies. Additional implications for Trojan orbits, and quantized effects in photon deflection were also noted.
\end{abstract}

Keywords. Dark energy, dark matter, galaxies: spiral, gravitation, hydrodynamics, large-scale structure of universe, minor planets, asteroids: general, planetary systems, relativistic processes

\section{References}

Bush, J. W. M. 2015, Annual Review of Fluid Mechanics, 47, 269

Couder, Y. \& Fort, E. 2006, Phys. Rev. Lett., 97, 154101

De Broglie, L. 1962, Cahiers de Physique, 16(147), 425

Munera, H. A., Buritica, D., Guzman, O., \& Vallejo, J. I. 1995, Revista Colombiana de Fisica, 27,215

Munera, H. A. \& Guzman, O. 1997, Foundations of Physics Letters, 10, 31

Munera, H. A. 2000, Momento (Revista de Fisica, Universidad Nacional de Colombia), 20, 1 